\section{Zur Metallverteilung in $\mathrm{SrFe}_{7} \mathbf{A l}_{5} \mathbf{o}_{19}$}

The Atomic Distribution in $\mathrm{SrFe}_{7} \mathrm{Al}_{5} \mathrm{O}_{19}$

\section{H. Pausch und Hк. Müller-Buschbaum Institut für Anorganische Chemie der Christian-Albrechts-Universität Kiel}

(Z. Naturforsch. 31 b, 1148 [1976]; eingegangen am 10. Mai 1976) Crystal Structure, Atomic Distribution

Single crystals of $\mathrm{SrO} \cdot 3.5 \mathrm{Fe}_{2} \mathrm{O}_{3} \cdot 2.5 \mathrm{Al}_{2} \mathrm{O}_{3}$ were prepared and investigated by $\mathrm{X}$-ray diffraction. The atomic distribution differs from former investigations of magnetoplumbite compounds.

Verbindungen der Zusammensetzung $\mathrm{SrO} \cdot 6 \mathrm{Me}_{2} \mathrm{O}_{3}$ $\left(\mathrm{Me}_{2} \mathrm{O}_{3}=\mathrm{Fe}_{2} \mathrm{O}_{3}, \mathrm{Al}_{2} \mathrm{O}_{3}, \mathrm{Ga}_{2} \mathrm{O}_{3}, \mathrm{Cr}_{2} \mathrm{O}_{3}\right)$ gehören zur Verbindungsklasse der Magnetoplumbite ${ }^{1}$. Zur gleichen Kristallstruktur gehören ebenfalls Verbindungen mit $\mathrm{CaO}$ bzw. $\mathrm{BaO}$ und gemischter Zusammensetzung im $\mathrm{Me}_{2} \mathrm{O}_{3}$-Anteil ${ }^{2}$ (z.B. :

$\left.\mathrm{BaO} \cdot 5 \mathrm{Fe}_{2} \mathrm{O}_{3} \cdot \mathrm{Al}_{2} \mathrm{O}_{3} ; \mathrm{BaO} \cdot 3 \mathrm{Fe}_{2} \mathrm{O}_{3} \cdot 3 \mathrm{Ga}_{2} \mathrm{O}_{3}\right)$. Bei den gemischt zusammengesetzten Magnetoplumbitvarianten interessiert die Verteilung der dreiwertigen Metalle auf die im Magnetoplumbit vorgegebenen Punktlagen (vgl. Tab. I).

Nach Untersuchungen von BERTAUT ${ }^{2}$ verteilen sich die dreiwertigen Metallionen nicht generell statistisch auf die in Tab. I angegebenen Punktlagen, sondern zeigen bei den aluminiumhaltigen Magnetoplumbitvarianten (z. B. $\mathrm{BaO} \cdot 5 \mathrm{Fe}_{2} \mathrm{O}_{3}$. $\mathrm{Al}_{2} \mathrm{O}_{3}$ ) eine teilweise geordnete Verteilung: $\mathrm{Al}$ in $2 \mathrm{a}, \mathrm{Fe}$ in $2 \mathrm{~b}, 4 \mathrm{f}_{\mathrm{I}}, 4 \mathrm{f}_{\mathrm{II}}$ und $2 \mathrm{Al}+10 \mathrm{Fe}$ in $12 \mathrm{k}$. Die zitierte Arbeit zeigt weiter, daß Al bevorzugt die Punktlage 2a geordnet, die Punktlage $12 \mathrm{k}$ partiell

Tab. I. Parameter für $\mathrm{Me}^{3+}$ im Magnetoplumbit (1). Raumgruppe $\mathrm{D}_{6 \mathrm{~h}}^{4}-\mathrm{P} 6_{3} / \mathrm{mmc}$.

\begin{tabular}{llll}
\hline Punktlage & $x$ & $y$ & $z$ \\
\hline $2 \mathrm{a}$ & 0,0 & 0,0 & 0,0 \\
$2 \mathrm{~b}$ & 0,0 & 0,0 & 0,250 \\
$4 \mathrm{f}_{\mathrm{I}}$ & 0,333 & 0,666 & 0,028 \\
$4 \mathrm{f}_{\mathrm{II}}$ & 0,333 & 0,666 & 0,189 \\
$12 \mathrm{k}$ & 0,167 & 0,334 & 0,892 \\
\hline
\end{tabular}

Sonderdruckanforderungen an Prof. Dr. Hк. MüLLER-BUSCHBAUM, Institut für anorganische Chemie der Universität Olshausenstraße 40-60, Haus 22 und 21 , D.2300 Kiel. statistisch besetzt. Bei steigendem Al-Anteil

$\left(\mathrm{BaO} \cdot 3 \mathrm{Fe}_{2} \mathrm{O}_{3} \cdot 3 \mathrm{Al}_{2} \mathrm{O}_{3}\right)$

wird zusätzlich $4 f_{\mathrm{I}}$ von Al mitbesetzt.

Bei der Untersuchung einer Verbindung der $\mathrm{Zu}$ sammensetzung $\mathrm{SrO} \cdot 3,5 \mathrm{Fe}_{2} \mathrm{O}_{3} \cdot 2,5 \mathrm{Al}_{2} \mathrm{O}_{3}$ (Gitterkonstanten: $a=571,4, c=2278,4 \mathrm{pm}$ ) wurde die bevorzugte Besetzung der Punktlage 2a durch Aluminium nicht gefunden. Die an 460 symmetrieunabhängigen Reflexen vorgenommene Einkristalluntersuchung (Vierkreisdiffraktometer PHILIPS PW 1100) zeigt bei einem Gütefaktor von $R=0,07$ die in Tab. II aufgeführte Atomverteilung ${ }^{3}$.

Der charakteristische Unterschied zur vergleichbaren Verbindung $\mathrm{BaO} \cdot 3 \mathrm{Fe}_{2} \mathrm{O}_{3} \cdot 3 \mathrm{Al}_{2} \mathrm{O}_{3}$ besteht darin, daß $\mathrm{Al}^{3+}$ in der hier untersuchten Verbindung nicht die scheinbar für $\mathrm{Al}^{3+}$ typische Punktlage $2 \mathrm{a}$ sondern die Punktlage $2 \mathrm{~b}$ besetzt. In $2 \mathrm{a}$ befindet sich $\mathrm{Fe}^{3+}$, was bei den Bariumverbindungen von Bertaut nie beobachtet wurde. Eine Betrachtung der Koordinationspolyeder $\left(4 \mathrm{f}_{\mathrm{I}}=\right.$ Tetraeder; $2 \mathrm{a}$, $4 \mathrm{f}_{\mathrm{II}}, 12 \mathrm{k}=$ Oktaeder, $2 \mathrm{~b}=$ trigonale Bipyramide) zeigt, daß die hier gefundene Metallverteilung $\left(\mathrm{Fe}^{3+}\right.$ im Oktaeder $(2 \mathrm{a}), \mathrm{Al}^{3+}$ in trigonaler Bipyramide $(2 \mathrm{~b})$ ) mit der Polyedergröße der Koordinationszahl und den Abständen harmoniert. $\mathrm{Fe}^{3+}$ (in 2a) besitzt $6 \mathrm{O}^{2-}$-Nachbarn im Abstand von $184 \mathrm{pm}, \mathrm{Al}^{3+}$ (in 2b) $3 \mathrm{O}^{2-}$-Nachbarn im Abstand $177 \mathrm{pm}$ und zwei deutlich weitere (Spitze und Fuß der trigonalen Bipyramide) mit 246 pm.

Tab. II. Parameter für $\mathrm{SrFe}_{7} \mathrm{Al}_{5} \mathrm{O}_{19}$, Raumgruppe $\mathrm{D}_{6 \mathrm{~h}}^{4}-\mathrm{P} 6_{3} / \mathrm{mmc}$.

\begin{tabular}{lllll}
\hline Atomart & Punktlage & $x$ & $y$ & $z$ \\
\hline $\mathrm{Sr}$ & $(2 \mathrm{~d})$ & 0,333 & 0,666 & 0,750 \\
$\mathrm{Fe}$ & $(2 \mathrm{a})$ & 0,0 & 0,0 & 0,0 \\
$\mathrm{Al}$ & $(2 \mathrm{~b})$ & 0,0 & 0,0 & 0,250 \\
$3 \mathrm{Fe} / \mathrm{Al}$ & $\left(4 \mathrm{f}_{\mathrm{I}}\right)$ & 0,333 & 0,666 & 0,027 \\
$\mathrm{Fe}$ & $\left(4 \mathrm{f}_{\mathrm{II}}\right)$ & 0,333 & 0,666 & 0,187 \\
$5 \mathrm{Fe} / 7 \mathrm{Al}$ & $(12 \mathrm{k})$ & 0,168 & 0,337 & 0,895 \\
$\mathrm{O}$ & $(4 \mathrm{e})$ & 0,0 & 0,0 & 0,142 \\
$\mathrm{O}$ & $(4 \mathrm{f})$ & 0,333 & 0,666 & 0,945 \\
$\mathrm{O}$ & $(6 \mathrm{~h})$ & 0,179 & 0,358 & 0,250 \\
$\mathrm{O}$ & $(12 \mathrm{k})$ & 0,146 & 0,292 & 0,050 \\
$\mathrm{O}$ & $(12 \mathrm{k})$ & 0,509 & 1,018 & 0,147 \\
\hline
\end{tabular}

Der Deutschen Forschungsgemeinschaft danken wir für die Unterstützung mit wertvollen Sachmitteln.

1 V. AdelsköLd, Ark. Kemi 12, 29 [1938].

2 E. F. Bertaut, A. Deschamps, R. Pauthenet u. S. Pickart, J. Phys. Rad. 2, 404 [1959].

3 H. Pausch, Dissertation Kiel 1976. 